\title{
Peierls stress in face-centered-cubic metals predicted from an improved semi-discrete variation Peierls-Nabarro model
}

\author{
Guisen Liu ${ }^{\mathrm{a}}$, Xi Cheng ${ }^{\mathrm{a}, \mathrm{b}}$, Jian Wang $^{\mathrm{c}}$, Kaiguo Chen ${ }^{\mathrm{d}}$, Yao Shen ${ }^{\mathrm{a}, *}$ \\ ${ }^{a}$ State Key Lab of Metal Matrix Composites, School of Materials Science and Engineering, \\ Shanghai Jiao Tong University, Shanghai 200240, China \\ ${ }^{\mathrm{b}}$ Department of Mechanical Engineering, Stanford University, CA94305, USA \\ ${ }^{\mathrm{c}}$ Department of Mechanical and Materials Engineering, University of Nebraska-Lincoln, \\ NE68588, USA \\ ${ }^{\mathrm{d} C e n t e r}$ for Compression Science, China Academy of Engineering Physics, Mianyang 621900, \\ China \\ * Corresponding author. E-mail: yaoshen@ sjtu.edu.cn
}

\begin{abstract}
In order to quantitatively predict Peierls stress, a semi-discrete variational Peierls-Nabarro model is improved by incorporating an additional gradient energy term into the energy functional. This gradient energy term is designed to effectively represent the influence of both the discreteness of atoms and the quick variations of the displacement profile in the dislocation core. Using face-centered-cubic metals as a model system for validation, we obtain a more accurate prediction of the displacement profile across the slip plane, and consequent precise Peierls stress, within few times the prediction from molecular dynamics calculations.
\end{abstract}

Keywords: Dislocation; Peierls stress; Slip profile; Peierls-Nabarro model; Gradient energy 
Dislocations are major carriers of plastic deformation in crystalline materials. Of particular interest is dislocation motion (velocity and mobility) in crystallites, which governs many of the macro-scale properties of crystallites during plastic deformation [1]. A critical resolved shear stress is defined to assess dislocation mobility, i.e., Peierls stress $\left(\tau_{\mathrm{p}}\right)[1]$ — the minimum external stress to move a straight dislocation without thermal activation. $\tau_{p}$ strongly depends on displacement fields around a dislocation (i.e. dislocation core), which is resulted from the atomic interactions among the dislocation region. Quantitative predictions of $\tau_{p}$ enhance our understanding of the generation and movement of dislocations amongst various materials with different slip systems [1]. The Peierls stress and the dependent dislocation core structures can be estimated through direct atomic simulations [2-4], these methods are however usually limited to some simple cases. Ab-initio density-functional theory (DFT) calculations provide high-precision results but with very high computational expense, and therefore cannot be applied to complicated cases. Molecular dynamics/molecular statics (MD/MS) simulations have very limited applications due to a lack of reliable and transferrable interatomic potentials.

Alternatively, more computationally efficient models have been developed by combining the continuum description of elastic medium with the discrete treatment of atoms in dislocation core region. Peierls [5] and Nabarro [6] firstly proposed such a hybrid model (the so-called PN model), which gave the analytical solution for the slip profile $u(x)$ and $\tau_{p}$. Joos and Duesbery [7] further improved the analytical derivation and obtained different expressions of $\tau_{p}$ for both wide and narrow dislocations. However, these PN models are considered as a conceptual tool because of its oversimplification and some inconsistency between the assumptions as discussed in [8-10].

In order to overcome these limitations [8-10], numerous extensions and modifications have been proposed. For instance, Vitek [11, 12] adopted the gradient of the generalized stacking fault energy ( $\gamma$-surface) to physically describe the atomic restoring force, instead of the simplified one-dimensional sinusoidal force law. The $\gamma$-surface can be obtained by 
atomistic or more accurate ab initio DFT calculations [13]. Also adopting $\gamma$-surface, Schoeck $[14,15]$ generalized the PN model for two dimensional cases via variational approach. Bulatov and Kaxiras [9] further corrected the inconsistency through a semi-discrete variational PN (SVPN) framework, in which the discrete summation of the misfit energy is employed for determination of both the slip profile and $\tau_{p}$, and the slip profile is allowed to vary during its interaction with the external applied stress. Shen and Cheng [10] extended the SVPN model by introducing a new method to calculate $\tau_{p}$ in a physically transparent way. In spite of these improvements, significant gap still exists between the $\tau_{p}$ predictions and experimental values, especially for dislocations in face-centered-cubic (FCC) metals. As a matter of fact, $\tau_{p}$ of those dislocations were predicted to be $10^{-4} \mu \sim 10^{-3} \mu$ ( $\mu$ being shear modulus) $[16,17]$ by the PN models, which were roughly two orders of magnitude higher than experimental measurements $10^{-6} \mu \sim 10^{-5} \mu[18]$.

Noticeably, quick variations of the slip profile (large displacement gradient) in the dislocation core [19, 20] considerably affects misfit energy and Peierls stress. Schoeck [19] suggested taking some averages methods in calculating misfit energy to account for the large displacement gradient, which can significantly lower Peierls energy and Peierls stress. However, difficulty in assessing the characteristic average distance makes it challenging to quantify the lowering magnitude. Instead of an empirical average distance, Miller et al [20] considered that the displacement gradient is caused by the nonlocal nature of atomic interactions, and its contribution to the misfit energy can be accounted for by incorporating a nonlocal kernel. Their nonlocal formulation was solved by calibrating the misfit energy with atomistic simulations, therefore greatly improved the misfit energy. However, core structure predicted by this approach was not improved much, but even deviated away from the atomistic simulations.

More recently, it was shown that the discrete nature of the lattice produces an extra term in the elastic energy that can smoothen the slip profile and reduce Peierls stress [21, 22]. Based on statics analysis, Wang $[21,22]$ found that in the stress balance equation (the Peierls 
equation), an additional force term proportional to the second derivative of the slip profile should stand up universally, which was however dropped off in continuum approximation. Equivalently, in energy functional form this additional force term can be cast into an integral of the square of the displacement gradient. When the discreteness effects were such considered in the elastic energy calculation, the modified model can smoothen the slip profile, predict wider core width and reduced $\tau_{p}[23,24]$. However, it is challenging to accurately predict $\tau_{p}$ due to the isotropic assumption and long-wavelength approximation in determining the proportional coefficients [22].

The purpose of this paper is to advance the SVPN framework $[9,10]$ by considering the effects of displacement gradient on misfit energy $[19,20]$ and discreteness effects on elastic energy $[21,22]$, and as a result, to further improve the prediction of Peierls stress. Consider a straight dislocation with Burgers vector $\mathbf{b}$ in a crystalline solid. Its core structure is described by the slip profile $\mathbf{u}(x)$, generally with three components $u_{k}(x)(k=1,2,3$, representing the direction of $x, y$ and $z$ axis), where $x$ denotes the coordinate perpendicular to the dislocation line. The equilibrium state of $\mathbf{u}(x)$ is then determined by minimizing the total energy with respect to $\mathbf{u}(x)$. The energy functional that describes a single dislocation is proposed to be:

$$
\begin{aligned}
& E_{\mathrm{dis}[}[\mathbf{u}(x)]=E_{\text {elastic }}+E_{\text {misfit }}+E_{\text {ext }}+E_{\text {gradient }}=\iint \frac{d \mathbf{u}\left(x^{\prime}\right)}{d x} \frac{\mathbf{H}}{x-x^{\prime}} \mathbf{u}(x) d x d x^{\prime} \\
& +\int \gamma[\mathbf{u}(x)] d x-\sum_{k} \int \tau_{k} \cdot u_{k}(x) d x+\lambda \int\|\nabla \mathbf{u}\|^{2} d x
\end{aligned}
$$

where the four terms in Eq.(1) correspond to the elastic energy stored in the two half-spaces $[8,25]$, the atomic misfit energy in the glide plane [25], the external work term $[9,10]$ and the additional gradient energy term we propose to consider the effects of displacement gradient $[19,20]$ and discreteness $[21,22] . \boldsymbol{H}$ is the Stroh tensor of the prelogarithmic energy factors [26], which depends on the elastic constants and the dislocation line, $\rho_{k}(x)=d u_{k} / d x$ denotes the dislocation density, $\gamma(\mathbf{u}(x))$ is the $\gamma$-surface [11], which can be obtained from DFT calculations or atomistic simulations by computing the energy penalty after shifting two atomic half spaces by a vector $\mathbf{u}(x)$ (see supplementary material), $\tau_{k}$ is the applied shear stress along the $k$ direction on the glide plane, and $\lambda$ is the gradient coefficient to be determined. 
Following the SVPN framework [9, 10], the slip profile $\mathbf{u}(x)$ could be expressed as a piecewise linear function interpolated between atomic sites $x_{i}$. Consider a full dislocation with Burgers vector $\vec{b}=1 / 2[\overline{1} 01]$ in FCC metals, which tends to dissociate into two Shockley partials separated by stacking faults [1] (see Fig. 1, we consider only two components of profile $u_{1}(x)$ and $u_{3}(x)$ for the planar core dislocation, and the vertical relaxation is simply accounted for in calculating $\gamma$-surface). The energy functional in Eq.(1) associated with the dislocation in Fig.1 can be recast in a discretized form:

$$
\begin{aligned}
& E_{\text {disl }}\left[\left\{u_{1}\left(x_{i}\right), u_{3}\left(x_{i}\right)\right\}\right]=\sum_{k=1,3} \sum_{i=1}^{N} \sum_{j=1}^{N} H_{k} x_{i j} \rho_{k}\left(x_{i}\right) \rho_{k}\left(x_{j}\right)+\sum_{i=1}^{N} \gamma\left[u_{1}\left(x_{i}\right), u_{3}\left(x_{i}\right)\right] \Delta x \\
& -\tau_{y z} \sum_{i=1}^{N}\left[u_{3}\left(x_{i}\right)+u_{3}\left(x_{i+1}\right)\right] \Delta x / 2+\lambda \sum_{k=1,3} \sum_{i=1}^{N}\left(\frac{u_{k}\left(x_{i+1}\right)-u_{k}\left(x_{i-1}\right)}{2 \Delta x}\right)^{2} \Delta x
\end{aligned}
$$

where $u_{k}\left(x_{i}\right)$ denotes the $k$-th component of the slip profile at atomic site $x_{\mathrm{i}} ; H_{1}=H_{11}, H_{3}=$ $H_{33}$ (see Table 1), $H_{13}=0$ (interaction between the screw and edge component vanishes for dislocations considered in this study); $\chi_{i j}=\frac{3}{2} \phi_{i+1, i} \phi_{j+1, j}+\psi_{i, j}+\psi_{i+1, j+1}-\psi_{i+1, j}-\psi_{i, j+1}$, with $\phi_{i, \mathrm{j}}=x_{i}-x_{j}, \psi_{i, j}=\frac{1}{2} \phi_{i, j}^{2} \ln \left|\phi_{i, j}\right|$; the displacement density $\rho_{k}\left(x_{i}\right)=\left[u_{k}\left(x_{i+1}\right)-u_{k}\left(x_{i}\right)\right] / \Delta x$; the interval of neighboring atomic sites $\Delta x=x_{i+1}-x_{i}(i=1, \cdots, N$, and $N \cdot \Delta x$ denotes the cut-off distance) is equal to $\sqrt{3} b / 2$ for screw dislocation and $b$ for edge dislocation; the $\gamma$-surface $\gamma\left[u_{1}, u_{3}\right]$ is calculated (see supplementary material) by MS simulation with embedded atom method potentials [27-29] when atoms relaxation is allowed in the $y$ direction; $\tau_{y z}$ is the applied glide stress (the non-glide stress $\tau_{y x}$ is zero); central difference of the profile is used to discretize $\nabla \mathbf{u}$, because it is conceptually more appropriate description of the "gradient", and numerically more accurate.

The equilibrium slip profile at any given applied stress $\tau_{y z}$ and gradient coefficient $\lambda$ could be determined by minimizing the total energy in Eq.(2). It is numerically solved by a simple steepest descent method, with the Volterra dislocation as the initial trial profile and the boundary nodes being fixed (i.e. $u_{3}\left(x_{N}\right)=b$ and $\left.u_{1}\left(x_{1}\right)=u_{1}\left(x_{N}\right)=u_{3}\left(x_{1}\right)=0\right)$. Fig.2a compares the equilibrium slip profiles of a screw dislocation in $\mathrm{Cu}$ at zero applied stress when $\lambda=0$ 
(previous SVPN model [10]) and $\lambda=0.252 \mu b$, where $\mu=\left(c_{11}-c_{12}+c_{44}\right) / 3$ is the shear modulus along <111> direction, and $c_{11}, c_{12}$ and $c_{44}$ are the elastic constants (see Table 1). The profiles calculated by MD simulations are also shown in Fig.2a. Obviously, the current model $(\lambda=0.252 \mu b)$ greatly improves the prediction for the dislocation core structure and brings the profile in excellent agreement of MD simulations, while the core structure predicted by the previous SVPN model [10] $(\lambda=0)$ is much narrower than that given by MD simulations.

The gradient coefficient $\lambda$ introduced in our model is numerically determined by calibrating the equilibrium slip profile at zero applied stress against that obtained by MD simulation, i.e.

$$
\lambda=\arg \min \left\{\sum_{i}\left(u_{3}^{\lambda}\left(x_{i}\right)-u_{3}^{M D}\left(x_{i}\right)\right)^{2}\right\}
$$

which is solved by minimizing the squares error between the predicted slip profile along Burgers vector by our model (as denoted by $u_{3}^{\lambda}\left(x_{i}\right)$ ) and that by MD simulations (as denoted as $\left.u_{3}^{M D}\left(x_{i}\right)\right)$. Table 2 gives $\lambda$ for the dislocations considered in this study. Different lambda for the screw dislocation and the edge dislocation is presumably because of their different elastic field.

Then Peierls stress is calculated by finding the critical applied stress at which the dislocation core is displaced significantly from its original location (on the order of several Burgers vector $[10,30])$. Variations in the slip profiles illustrated in Fig.2b indicate that $\tau_{p}$ predicted by the current model is $13 \mathrm{MPa}$, which is about one magnitude lower than that predicted by SVPN model $\left(\lambda=0, \tau_{p}=117 \mathrm{MPa}\right.$, see Table 2$)$. The result is rational considering the wider dislocation width (about $1 b$ ) computed by the modified model (see Fig. 2a). Besides, accuracy of $\tau_{p}$ is greatly improved, reducing from 40 times higher of MD prediction (2.9MPa, see Table 2), to within 4 times of MD calculations, and therefore much closer to experimental results [19].

The proposed model is further evaluated by applying to dislocations in more cases, 
including the edge dislocation in $\mathrm{Cu}$, and edge and screw in $\mathrm{Ag}$ and $\mathrm{Al}$. Table 2 compares $\tau_{p}$ calculated by the current model, previous SVPN model $(\lambda=0)[10]$ and MD simulations. The improvement in calculating $\tau_{p}$ is clearly shown in all six cases: while previous SVPN model gives $\tau_{p}$ that are 30 70 times larger than that by MD simulation (except the screw dislocation in $\mathrm{Al}$ ), the proposed model predicts $\tau_{p}$ much closer to MD results, reducing the overestimation to only $1 \sim 4$ times. The less than 4 times overestimation of $\tau_{p}$ could be considered quite good prediction in PN model. $\tau_{p}$ for the dislocations in $\mathrm{Al}$ are also compared with the computations in the double-counting scheme of SVPN model [31], which predicts $\tau_{p}$ about 6 times MD calculation for screw and 1 time for edge, while the proposed model predicts 3 times for both cases, indicating a reduced largest deviation. Overall, the modified SVPN model greatly improves the prediction of $\tau_{p}$ for dislocations in FCC metals.

The performance of the proposed model in describing the variation in dislocation core during its movement over the Peierls barrier is evaluated by comparing with MD calculations. The slip profiles along the minimum energy path (MEP) can be obtained by using the nudged elastic band method $[32,33]$ (see supplementary materials). Variations of stacking fault width (SFW), defined as the distance between the two partial dislocations (e.g. $\left.x\right|_{u_{3}=0.75 b}-\left.x\right|_{u_{3}=0.25 b}$ ), are considered the key factor characterizing the variations of dislocation core structure. For example, the variations of SFWs for a screw dislocation in $\mathrm{Cu}$ are shown in Fig. 3, and the amplitude of the oscillations of SFW is smaller than $\Delta x$, the upper limit of the variations. The modified model captures the same trend as MD simulations: the spacing decreases and then recovers to the equilibrium distance. However, the previous SVPN model gave the opposite trend. This finding implies that the proposed model improves not only the prediction of $\tau_{p}$, but also the variations of core structure during dislocation motion.

The calibration of gradient coefficient $\lambda$ relies heavily on dislocation profiles in the core region rather than the whole extension. Fig. 4 shows the calculated $\lambda$ that are obtained under different ranges in dislocation core considered for the least square problem described by Eq.(3). The results show that $\lambda$ rapidly converges after the calibration radius within the core 
increases beyond $3 b$. The results are consistent with the physical interpretation of $\lambda$, which partly describes the quick variations of the slip profile in the dislocation core, and is expected to influence the atomic arrangement within a narrow window around the dislocation core.

A potential advantage of the proposed model is that it may be applied to predict $\tau_{p}$ for structures whose interatomic potentials are not available, as long as their dislocation core structures can be characterized experimentally with sufficient accuracy. Considering the rapid development of advanced experimental techniques such as aberration corrected high resolution transmission electron microscopy, characterization of dislocation core with sufficient accuracy could be expected in the near future [34]. In this scenario, the gradient coefficient $\lambda$ shall be determined by calibrating the predicted profile against the ones measured experimentally, and the $\gamma$-GSF surface would be computed via DFT calculations [13], therefore the proposed model has the potential to be applied to more complex crystal structures.

In summary, we have proposed an enhanced SVPN model by incorporating a gradient energy term expressed in terms of the gradient of the slip profile. By appropriately calibrating the proposed model against the MD simulation on dislocation core structure, the model is capable of accurately predicting $\tau_{p}$ for dislocations in FCC structures. The results imply that the displacement gradient [19, 20] and discreteness effects [21, 22] play an important role in determining the dislocation profile and Peierls stress. It is hoped that the current work can widen the applicability of PN models in more complex crystal structures.

The research was funded by the National Science Foundation of China project 51471107, and CCS project Grant No.YK2015-0202002. Supercomputer of SJTU, $\pi$, is also acknowledged for the MD calculations. Helpful discussions with Professors Wei Cai and Ju Li are greatly appreciated. 


\section{References}

[1] J. P. Hirth, J. Lothe, Theory of Dislocations, second ed.,Wiley, New York, 1982.

[2] S.K. Yadav, R. Ramprasad, A. Misra, X.Y. Liu, Acta Mater. 74 (2014) 268-277.

[3] L. Ventelon, F. Willaime, E. Clouet, D. Rodney, Acta Mater. 61(2013) 3973-3985.

[4] S. Groh, E. Marin, M. Horstemeyer, D. Bammann, Model. Simul. Mater. Sci. Eng. 17 (2009) 075009.

[5] R. Peierls, Proc. Phys. Soc. 52 (1940) 34-37.

[6] F. Nabarro, Proc. Phys. Soc. 59 (1947) 256.

[7] B. Joos, M. Duesbery, Phys. Rev. Lett. 78 (1997) 266-269.

[8] G. Schoeck, Mater. Sci. Eng. A 400 (2005) 7-17.

[9] V.V. Bulatov, E. Kaxiras, Phys. Rev. Lett. 78 (1997) 4221.

[10] Y. Shen, X. Cheng, Scripta Mater. 61 (2009) 457-460.

[11] V. Vitek, Philos. Mag. 18 (1968) 773-786.

[12] V. Vitek, F. Kroupa, Philos. Mag. 19 (1969) 265-284.

[13] G. Lu, N. Kioussis, V.V. Bulatov, E. Kaxiras, Phys. Rev. B 62 (2000) 3099.

[14] G. Schoeck, Philos. Mag. A 69 (1994) 1085-1095.

[15] G. Schoeck, Acta Mater. 45 (1997) 2597-2605.

[16] G. Schoeck, Mater. Sci. Eng. A 558 (2012) 162-169.

[17] B. von Sydow, J. Hartford, G. Wahnström, Comp. Mater. Sci. 15 (1999) 367-379.

[18] Y. Kamimura, K. Edagawa, S. Takeuchi, Acta Mater. 61 (2013) 294-309.

[19] G. Schoeck, Phys. Rev. Lett. 82 (1999) 2310.

[20] R. Miller, R. Phillips, G. Beltz, M. Ortiz, J. Mech. Phys. Solids, 46 (1998) 1845-1867.

[21] S.F. Wang, Phys. Rev. B 65 (2002) 094111.

[22] S.F.Wang, J. Phys. A: Math. Theor. 42 (2009) 025208.

[23] X. Wu, Acta Mech. Solida Sin. 21 (2008) 403-410.

[24] R. Wang, S. Wang, X. Wu, Phys. Scripta, 83 (2011) 045604.

[25] V.V. Bulatov, W. Cai, Computer simulations of dislocations, Oxford University Press, 2006.

[26] D. Bacon, D. Barnett, R.O. Scattergood, Prog. Mater. Sci. 23 (1980) 51-262.

[27] Y. Mishin, M. Mehl, D. Papaconstantopoulos, A. Voter, J. Kress, Phys. Rev. B 63 (2001) 224106.

[28] X.Y. Liu, F. Ercolessi, J.B. Adams, Model. Simul. Mater. Sci. Eng. 12 (2004) 665-670.

[29] P. Williams, Y. Mishin, J. Hamilton, Model. Simul. Mater. Sci. Eng. 14 (2006) 817-833.

[30] X. Cheng, Y. Shen, L. Zhang, X. Liu, Philos. Mag. Lett. 92 (2012) 270-277.

[31] G. Lu, N. Kioussis, V.V. Bulatov, E. Kaxiras, Philos. Mag. Lett. 80 (2000) 675-682.

[32] G. Henkelman, H. Jónsson, J. Chem. Phys. 113 (2000) 9978.

[33] G. Henkelman, B.P. Uberuaga, H. Jónsson, J. Chem. Phys.113 (2000) 9901.

[34] M.J. Mills, N.L. Baluc, P.M. Sarosi, Microsc. Res. Tech. 69 (2006) 317-329. 
Figure 1. (a) Schematic illustrations of full screw (left) and edge (right) dislocations with Burgers vector $\vec{b}=1 / 2[\overline{1} 01]$ on (111) plane in FCC structure. In order to achieve the minimum energy state, the full dislocations tend to decompose into two Shockley partial dislocations. (b-c) Illustrations of the stationary slip profiles along the slip directions for the screw (b) and edge dislocations (c) on (111) plane. Screw dislocation usually possesses a narrower width comparing to edge dislocation.

Figure. 2. (a) Predicted slip profiles $u_{1}(x)$ and $u_{3}(x)$ of a screw dislocation in $\mathrm{Cu}$ that are calculated using SVPN $(\lambda=0)[10]$, modified SVPN model $(\lambda=0.252 \mu b)$ and MD simulations at zero applied stress. (b) Slip profiles of the screw dislocation in $\mathrm{Cu}$ predicted by the modified SVPN model with increasing applied glide stress $\tau_{y z} \cdot \tau_{p}$ is determined as the critical applied glide stress which causes significant displacement of dislocations from the original position. When the applied stress is below $\tau_{p}$, the predicted slip profile slightly adjusts its configuration.

Figure. 3. Variation of SFW, the distance between the two Shockley partials, along the minimum energy path of a screw dislocation moves in $\mathrm{Cu}$.

Figure. 4. Variations of gradient coefficient $\lambda$ computed under different ranges in dislocation core considered for the least square problem described by Eq.(3). 
Table 1. Elastic constants $c_{i j}$, shear modulus $\mu_{(111)}$ calculated by MS simulations for $\mathrm{Cu}, \mathrm{Ag}$ and Al. Stroh tensor $H_{i j}$ for screw and edge dislocations calculated by anisotropic theory [26]. All quantities are in the unit of GPa.

\begin{tabular}{|c|c|c|c|c|c|c|c|c|}
\hline & \multirow{2}{*}{$c_{11}$} & \multirow{2}{*}{$c_{12}$} & \multirow{2}{*}{$c_{44}$} & \multirow{2}{*}{$\mu_{(111)}$} & \multicolumn{2}{|c|}{ screw } & \multicolumn{2}{|c|}{ edge } \\
\hline & & & & & $H_{11}$ & $H_{33}$ & $H_{11}$ & $H_{33}$ \\
\hline $\mathrm{Cu}$ & 169.9 & 122.6 & 76.2 & 41.2 & 6.05 & 3.38 & 3.55 & 5.94 \\
\hline $\mathrm{Ag}$ & 124.2 & 93.9 & 46.4 & 25.6 & 3.92 & 2.11 & 2.21 & 3.85 \\
\hline $\mathrm{Al}$ & 118.9 & 62.9 & 33.0 & 29.7 & 3.68 & 2.42 & 2.42 & 3.68 \\
\hline
\end{tabular}

Table 2. Peierls stress $\tau_{p}$ of the screw and edge dislocation in $\mathrm{Cu}, \mathrm{Ag}$ and $\mathrm{Al}$ calculated by the modified SVPN model, MD simulations and the previous SVPN model $(\lambda=0)[10]$. The first column is the computed gradient coefficient $\lambda$ based on Eq.(3).

\begin{tabular}{cccccccc}
\hline & & \multicolumn{2}{c}{ Modified model } & & \multicolumn{2}{c}{$\tau_{p}(\mathrm{MPa})$} \\
\cline { 3 - 4 } \cline { 7 - 8 } $\mathrm{Cu}$ & & $\lambda(\mu b)$ & $\tau_{p}(\mathrm{MPa})$ & & MD & $\lambda=0$ \\
\hline \multirow{2}{*}{$\mathrm{Ag}$} & screw & 0.252 & 13 & & 2.9 & 117 \\
& edge & 0.566 & 4 & & 1.3 & 69 \\
& screw & 0.219 & 10 & & 3.1 & 125 \\
& edge & 0.309 & 1 & & 0.8 & 54 \\
\multirow{2}{*}{$\mathrm{Al}$} & screw & 0.141 & 125 & & 37 & 275 \\
& edge & 0.609 & 9 & & 2.6 & 119 \\
\hline
\end{tabular}


(a) $\boldsymbol{\Psi}^{3}$ or $z[\overline{1} 01] / / \vec{b}$

on (111) plane: $\vec{b} \rightarrow \vec{b}_{1}+\vec{b}_{2}+$ stacking faults
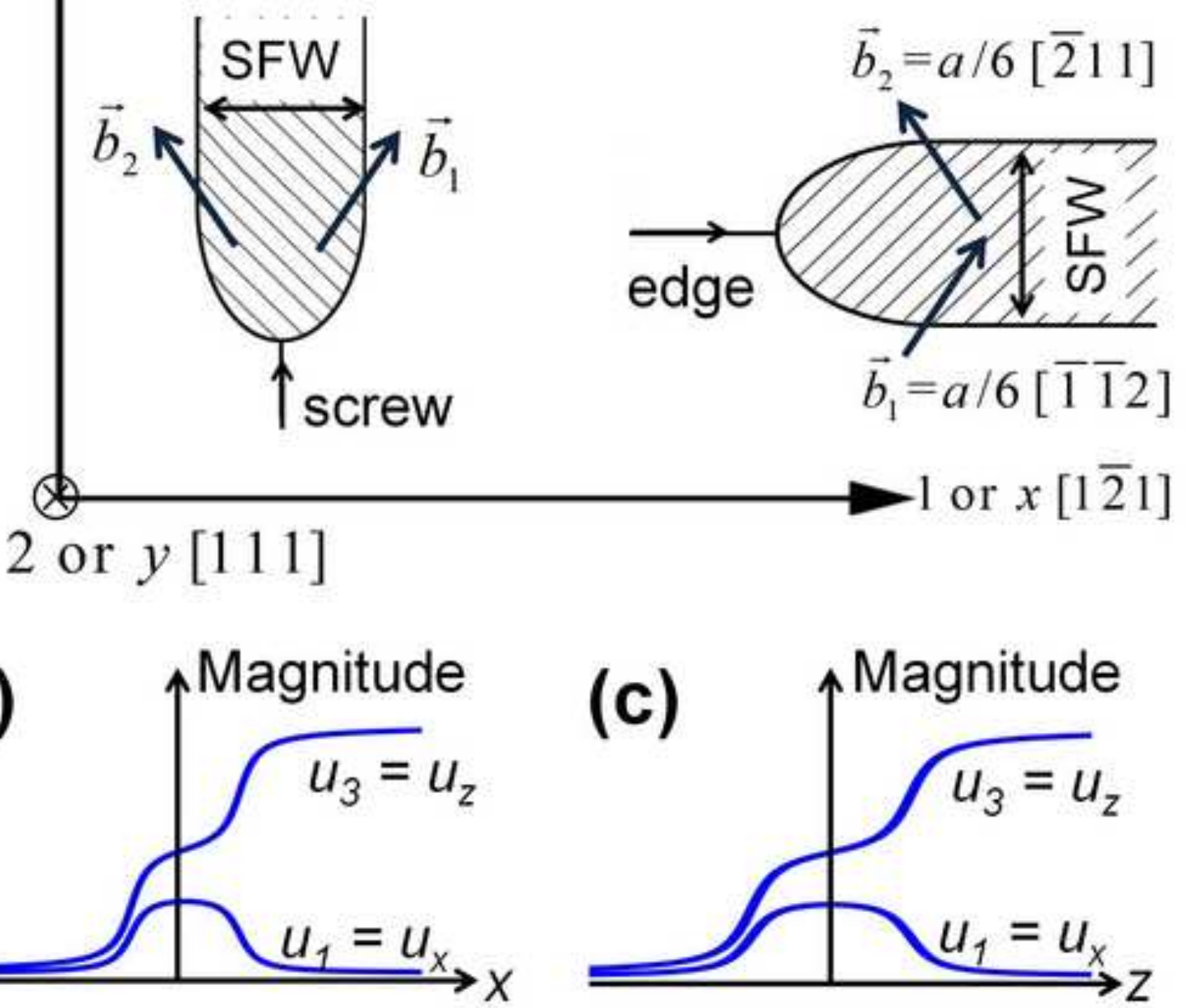

(b)

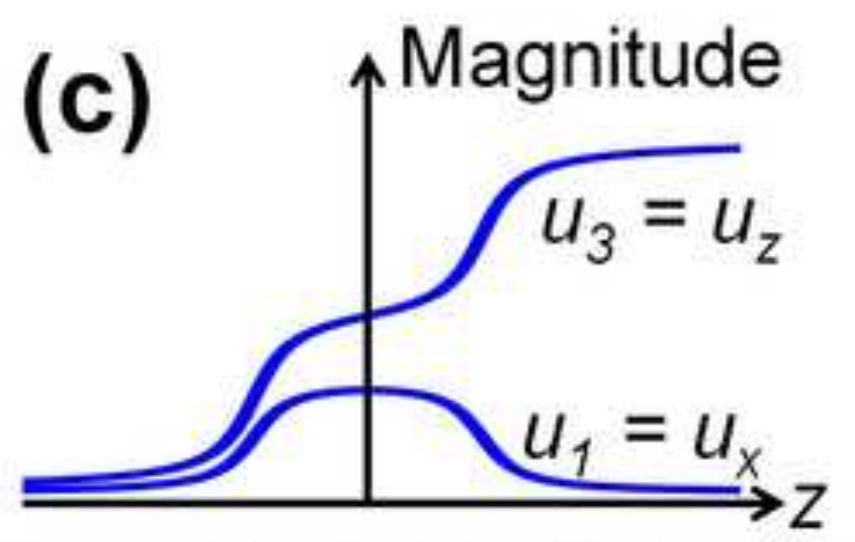

Dissociated screw dislocation Dissociated edge dislocation 

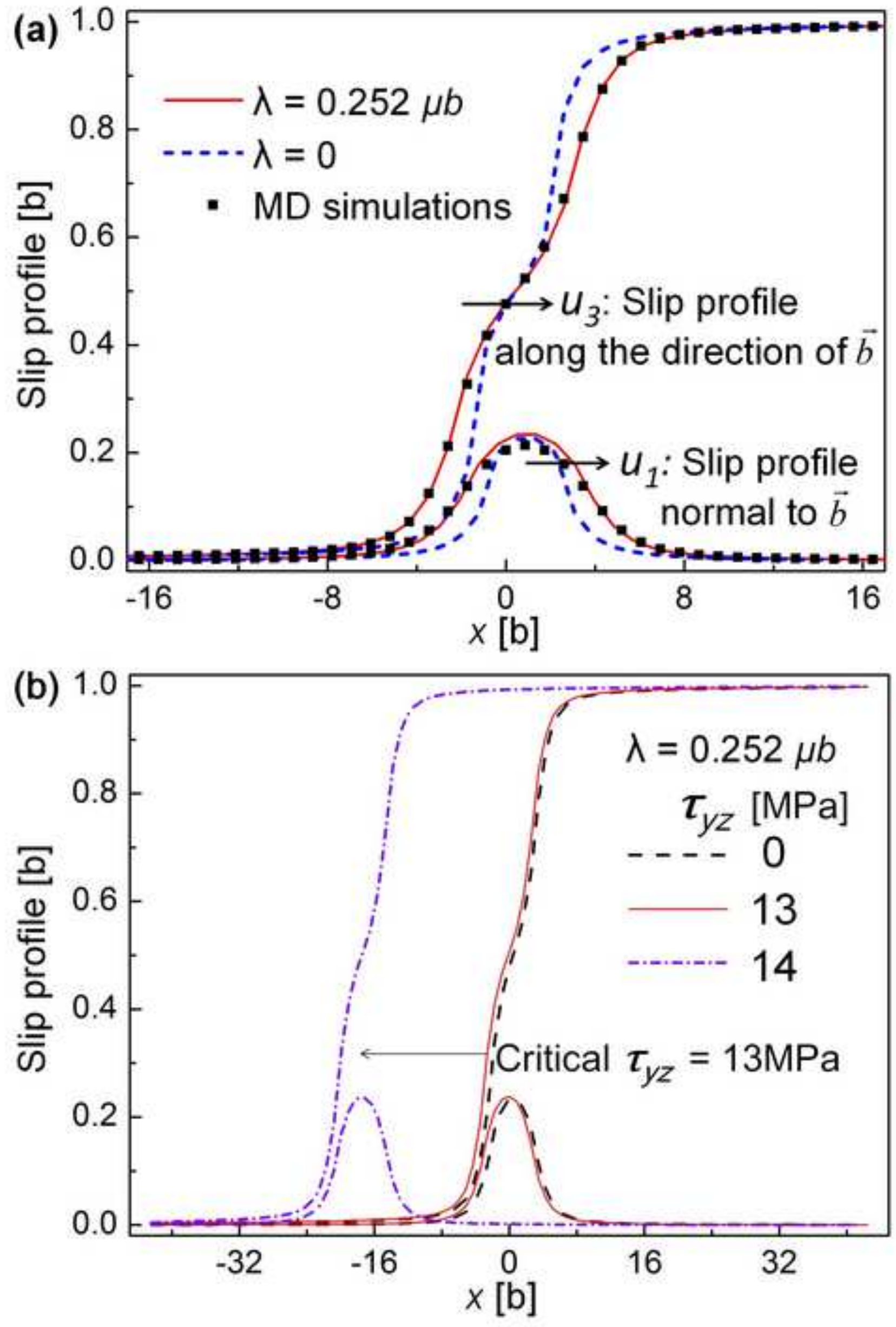


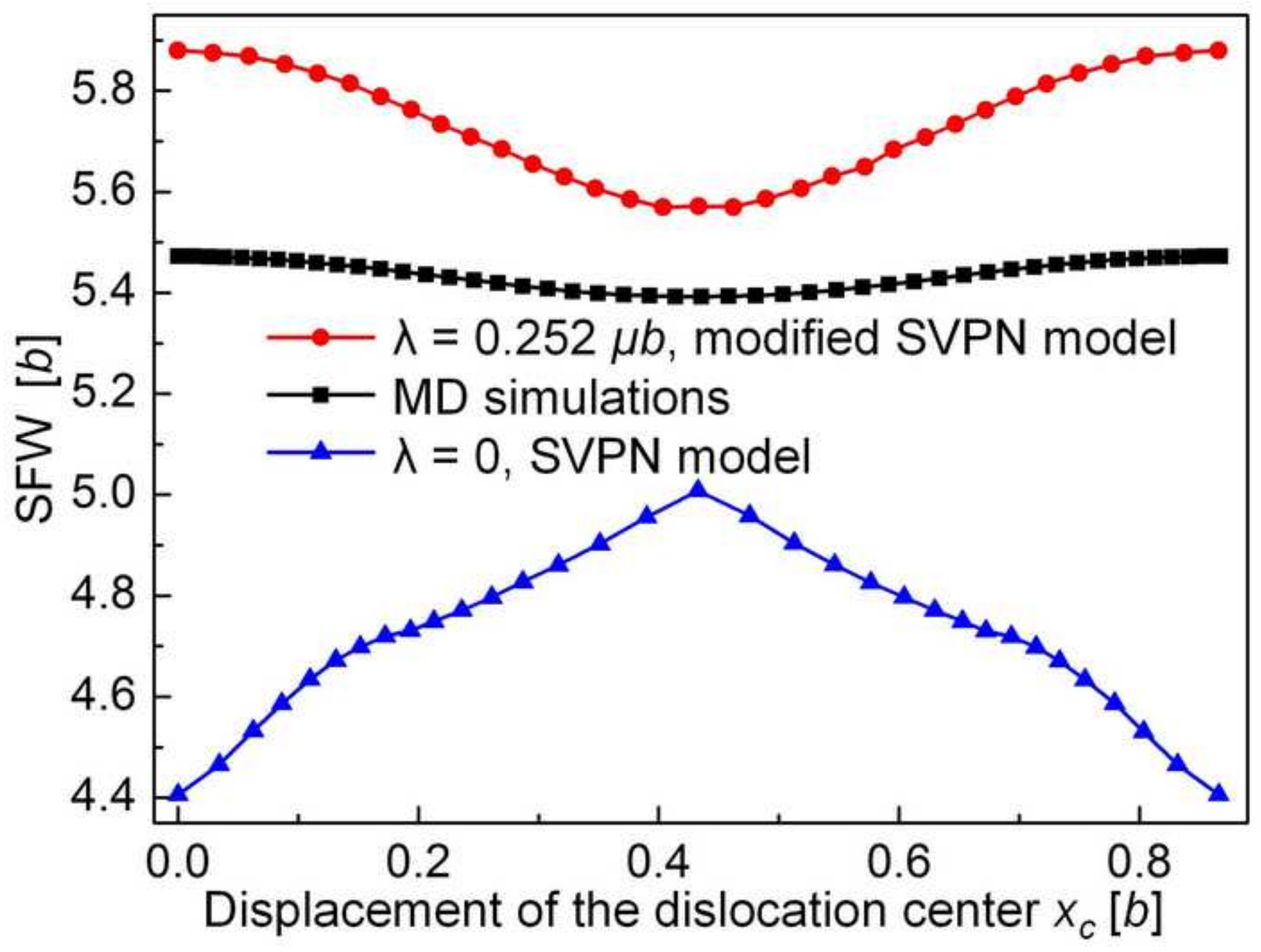



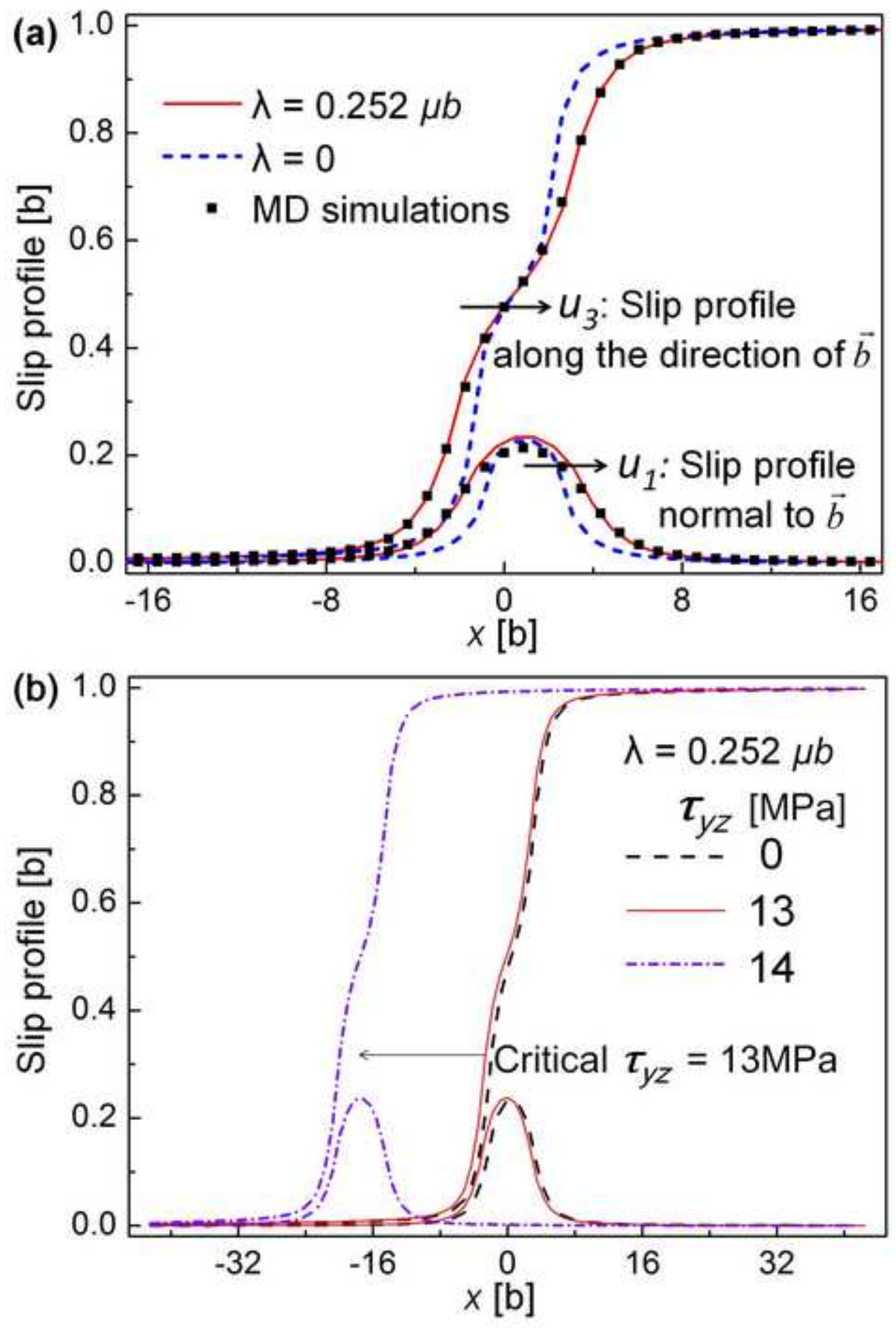